Enfin, pour que l'échelle des couples reste aussi constante, on prendra pour la détermination graphique du couple une parallele située à une distance constante de $\mathrm{OB}$, au lieu de se servir de la droite $A V$ (fig. 2) qui ici se déplace párallèlement à $\mathrm{OB}$, à mesure que $R$ varie.

Extension de la méthode aux autres modes d'excitation. - Dans les raisonnements précédents, nous n'avons fait aucune restriction sur la forme de la caractérisque à vide; nous avons supposé seulement que $e_{0}$ ne dépendait que de $I$ pour une vitesse $n_{0}$ déterminée. La méthode s'appliquera donc également à l'étude de la marche à potentiel constant dans les cas suivants :

- Moteurs à excitation séparée ou en dérivation. La caractéristique est alors une droite parallèle à $O B$; le courant s'annule pour une valeur finie de la vitesse, puis change de sens ; c'est une propriété bien connue du moteur en dérivation sur laquelle nous n'insisterons pas.

$2^{\circ}$ Moteurs à excitation composée additionnelle. On a affaire ici à la combinaison des deux excitations série et dérivation ; on peut donc obtenir la courbe d'excitation en déplaçant l'axe OE (fig. I) vers la droite d'une quantité correspondant aux ampères-tours en dérivation, la courbe OM restant fixe. La courbe alors ne passe plus par l'origine et il en résulte que, comme pour l'excitation en dérivation, le courant s'annule pour une valeur finie de la vitesse.

30 Moteurs à excitation composée différentielle. Dans ce cas, l'enroulement-série est démagnétisant ; cette disposition s'emploie, comme on le sait, pour maintenir la vitesse de rotation sensiblement constante, malgré de fortes rariations de charge. La courbe d'excitation s'obtiendra évidemment en déplaçant à gauche l'axe $\mathrm{OE}$, comme dans le cas précédent, mais on considèrera seulement, dans la marche en moteur, la portion à gauche de l'axe OE; la longueur $I_{0}$ sera portée à gauche et le sens des vitesses croissantes sera de gauche à droite.

Comme cela était évident a priori, le flux s'annule pour une certaine valeur de $I$ et est maximum pour $I=0$. L'étude de ce genre d'excitation est très intéressante; la marche du moteur change tout à fait d'allure suivant que $I_{0}$ est plus grand ou plus petit que la valeur du courant $I$ annulant le flux. Nous laissons au lecteur le soin d'étudier ces divers cas qui ne présentent aucune difficulté.

\section{J. Bethenod,} Ingénieur E. C. L.

\section{$\longrightarrow$}

\section{NOTE SUR L'USINE DE RHEINFELDEN}

\section{TRANSPORT DE FORCE ET ELECTROCHIMIE}

Nous avons cru intéressant de dire ici quelques mots sur cette usine qui réalise le problème de la meilleure utilisation des chutes d'eau, indiquée par M. Côte dans son article: "Transport de Force et Electrochimie »(I). A la théorie de M. Côte, à savoir que ces deux utilisations simultanéẹs de la houille blanche ne sauraient convenir

(1) Voir La Houille Blanche, $\mathbf{2}^{\mathrm{e}}$ année, $\mathrm{n}^{\mathrm{o}}$ I I (novembre 1903). qu'aux hautes chutes des Alpes, nous opposons l'exemple suivant d'une usine alimentée par une basse chute et qui fait à la fois, dans d'excellentes conditions économiques, du transport de force et de l'électrochimie. Il convient, toutefois, de remarquer que cette usine se trouve dans une situation exceptionnelle sous le rapport du prix de revient du cheval-an, ce qui lui a permis d'établir des tarifs très rémunérateurs pour la partie de son énergie qu'elle vend sous forme de force motrice et d'éclairage, et de livrer à bon marché le courant pour l'électrochimie.

Cette situation tient d'abord à ce fait que le Rhin, alimenté l'été par des glaciers et l'hiver par les régions basses de la Suisse et de la Forêt Noire, présente un régime régularisé, participant à la fois du régime alpestre et de celui des rivières de plaines, ensuite à ce que, grâce au cours rapide du fleuve en cet endroit, on a pu aménager à peu de frais un gros débit. Il ne s'agit donc pas ici d'une usine à puissance très variable n'utilisant que ses "chevaux périodiques "ou à peu près, à l'électrochimie, mais bien d'une usine à puissance sensiblement constante qui trouve, néanmoins, intérêt à utiliser une partie de son énergie, de ses " chevaux permanents », résultant d'un gros débit sous basse chute, à des opérations électrochimiques et cela parce que les dites opérations lui assurent l'emploi de chevaux de 24 heures. Si cette solution de la meilleure

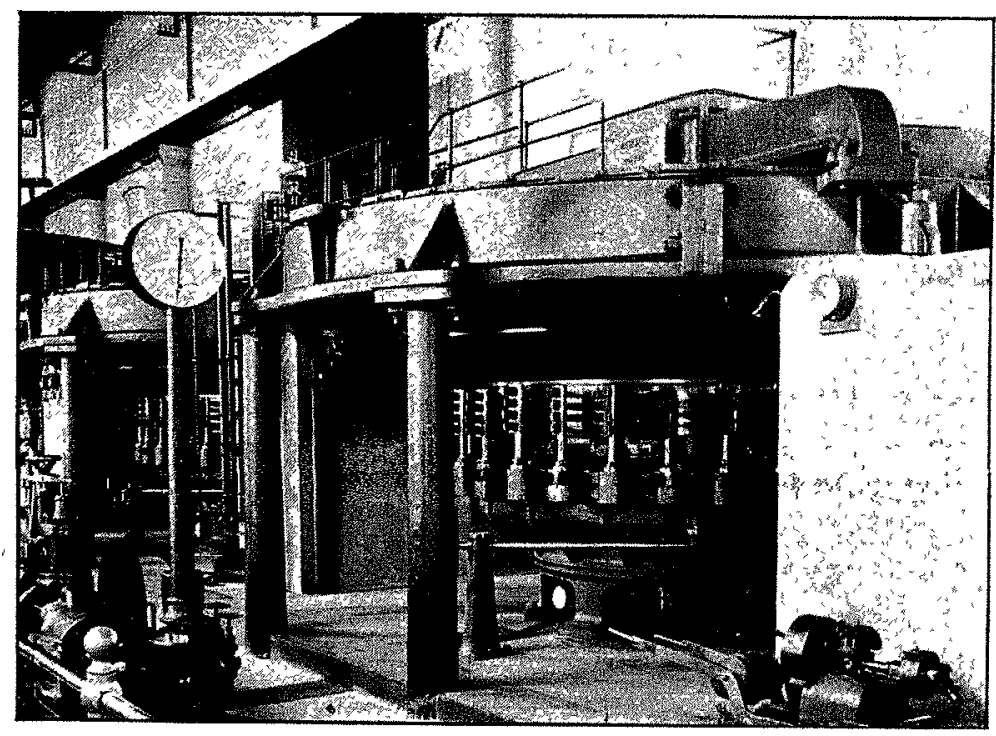

Vue d'une génératrice à courant continu.

utilisation des chutes n'est pas générale, ce cas particulier - et il doit s'en présenter d'autres - n'en offre pas moins beaucoup d'intérêt.

Aménagement de la chute. - Cette usine est située sur la rive badoise du Rhin, à deux kilomètres au-dessus de la petite ville de Rheinfelden. Elle est alimentée par une chute obtenue au moyen d'un barrage établi en travers du fleuve, à un kilomètre en amont. Ce barrage de $197^{\mathrm{m}} 7^{\circ}$ de longueur formant déversoir et muni de deux échelles à poissons, relève le plan d'eau de la quantité voulue pour assurer la prise du canal de dérivation, laquelle est commandée par huit grandes vannes à glissières laissant passer aux basses eaux une lame d'eau de I mètre d'épaisseur sur 22 mètres de largeur. Le canal, large de 80 mètres, présente une pente régulière de 60 centimètres sur la totalité de sa longueur qui est de 1 ooo mètres. La profondeur de l'eau y varie de $3^{\mathrm{m}} 7 \mathrm{o}$ en basses eaux à $5 \mathrm{~m} 30$ en hautes eaux. Il n'est séparé du Rhin que par une digue en maçonnerie. 
Entre le barrage et l'entrée du canal se trouve une chambre de décantation dont les vannes de purge permettent la chasse des apports dans le fleuve.

A l'extrémité aval du canal, une grille formée de barreaux de 7 mètres de longueur et espacés de 35 millimètres règne en avant de l'usine sur une longueur de 165 mètres. Celleci affecte en plan la forme d'un long rectangle dont la grande base est en prolongement de l'un des murs du canal, c'est-à-dire parallèle au Rhin. Sa façade principale vue de la rive suisse, a certaine ressemblance avec celle de l'usine de Jonage, mais son architecture en est plus sévère.

Du canal, l'eau passe dans vingt chambres munies chacune de deux portes tournantes en fer et qui renferment deux turbines du type Francis calées sur un même arbre vertical et faisant ensemble au minimum 840 chevaux, ce qui donne pour toute l'usine une puissance minima de 16800 chevaux. La disposition de ces moteurs rappelle tout à fait celle bien connue de l'usine de Chèvres.

La régulation de la vitesse des turbines s'effectue à l'aide de vannes cylindriques commandées par un régulateur à servomoteur employant l'huile comprimée à 25 kilos. Ces moteurs sont supportés par des crapaudines annulaires d'un diametre extérieur de $800 \mathrm{~m} / \mathrm{m}$, placées à la hauteur du plancher des machines et recevant l'huile sous la pression de 25 kilos. Dans ce but, chaque turbine actionne une pompe différentielle à huile, à simple effet, et toutes les pompes travaillent en batterie sur la même conduite envoyant thuile dans les crapaudines et les servo-moteurs des régulateurs. Au retour de ces appareils, le lubréfiart subit un double filtrage et revient aux pompes.

Equipement électrique. - Les vingt génératrices électriques à axe vertical sont toutes couplées directement aux turbines comme à Jonage et à Chèvres. L'usine comprend :

io Pour l'Electrochimie : Six génératrices à courant continu donnant go volts et 6000 ampères à la vitesse de 55 tours par minute. Leur courant est utilisé pour la fabrication de l'aluminium et du carbure de calcium par la succursale badoise à Rheinfelden de la «Aluminium industrie Aktiengeselleschaft Neuhausen »;

Deux génératrices à courant continu à 155 volts èt 4000 ampères tournant à 68 tours par minute, qui aljmentent une fabrique de soude électrolytique;

Quatre génératrices de 130 à 140 volts et 4500 ampères à la vitesse de 68 tours par minute, fournissant du courant continu à l'usine électrochimique badoise de la a Greisheim Electron " située à proximité ( 150 mètres environ) qui fabrique de l'hypochlorite de chaux et du carbure de calcium;

Le courant de ces douze machines est employé directement aux opérations électrochimiques par les usines aux: quelles il est destiné.

$2^{\circ}$ Pour la transport d'Énergie : Trois génératrices triphasées donnant 6800 volts et 59 ampères à la vitesse de 55 tours par minute, du type à fer tournant - type aujourd'hui abandonné à cause de son prix élevé.
Cinq génératrices triphasées de 6800 volts et $59 \mathrm{am}$. pères tournant à 68 tours par minute, avec inducteur tournant.

Le courant de ces huit machines, distribué dans la région sous forme de force motrice et de lumière, est transformé comme il séra dit plus loin.

L'excitation des alternateurs est obtenue au moyen de deux groupes moteurs-générateurs dont les moteurs triphasés sont alimentés par du courant pris à 6800 volts sur les barres collectrices et transformé à 500 volts; la tension d'excitation est de 155 à r 70 volts. En cas d'accident à ces groupes, de mème que pour la mise en marche des alterna-

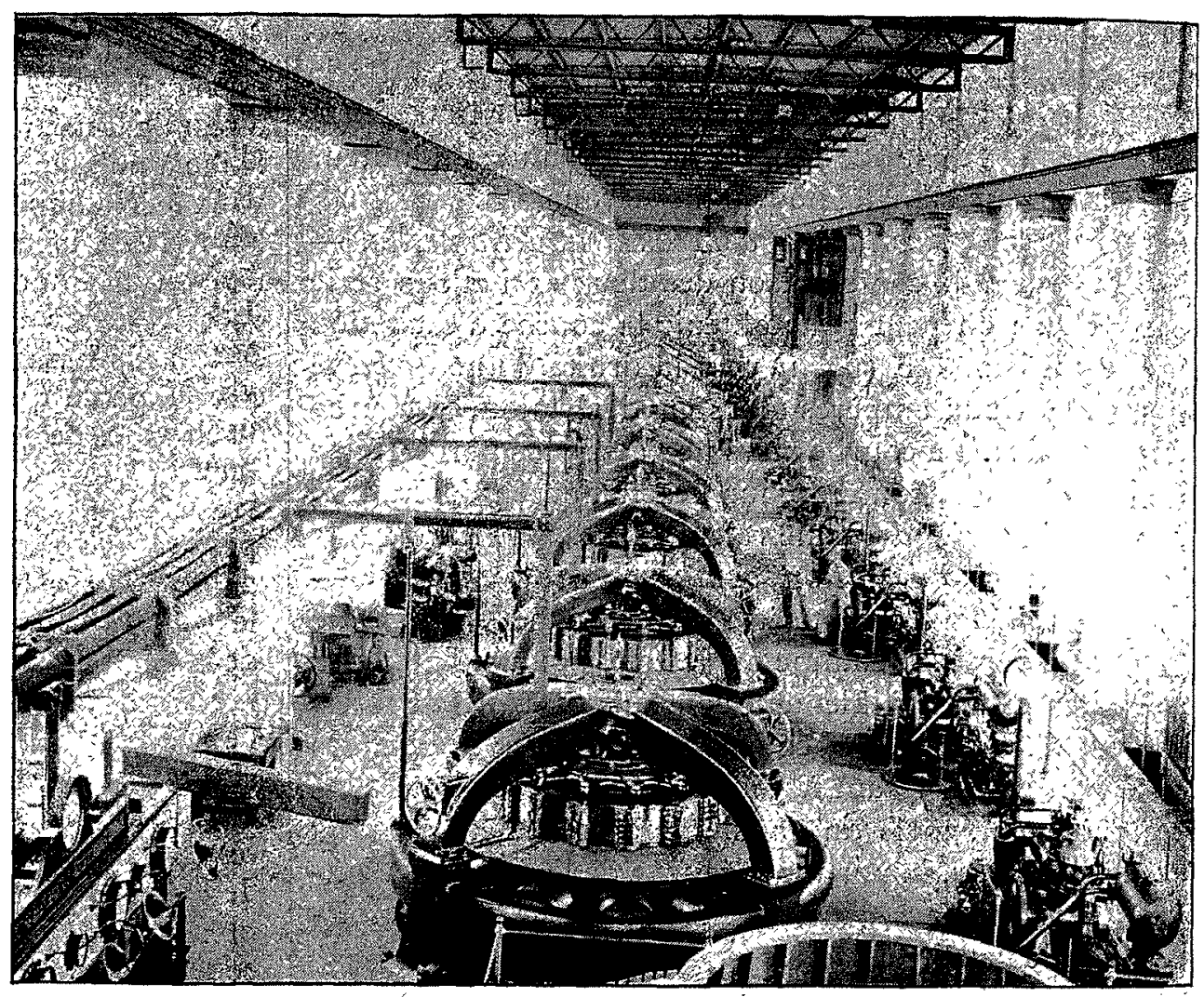

Vue générale de la salle des machines pour l'électrochinie.

teurs, on dispose pour l'excitation de trois dès machines a courant continu de l'électrochímie.

Les connexions des génératrices triphasées sont faites dans la partie centrale de l'usine et le tableau de distribution se trouve à la hauteur voulue pour que de sa plateforme on puisse surveiller toutes les machines.

Tous les alternateurs travaillent en parallèle sur les barres omnibus qui sont rangées par groupes, de telle manière qu'on puisse faire toutes réparations nécessaires sans déranger la marche de cette partie de l'usine. Les barres collectrices auxiliaires, disposées près des premières, peuvent recevoir le courant de deux alternateurs ou être montées en parallèle avec les barres principales. Les conducteurs et les câbles souterrains peuvent à volonté être mis sur les barres principales ou sur les barres auxiliaires, ce qui permet, à la suite d'une réparátion, d'essayer et de mettre en service séparément chaque conducteur, ainsi que de séparer du réseau de lumière le réseau de force qui est sujet à des variations de charges considérables.

Groupe a vapeur dé réserve. - En plus des machines précédemment indiquées, l'usine possède un groupe électrogène à vapeur, de réserve, installé dans un bâtinent 
spécial. Il est formé d'une turbine à vapeur Brown-BoveriParson's, accoupléc directement à une génératrice triphasée de $1400 \mathrm{KVA}$ donnant 6800 volts à la vitesse de 1500 tours à la minute.

La turbine à vapeur fonctionne ordinairement avec condensation, mais elle peut marcher à échappement libre. La vapeur lui est fournie surchauffée et à I 2 kilos I $/ 2$ de pression par une batterie de quatre chaudières, système Guilleaume, ayant chacune $283 \mathrm{~m}^{2}$ de surface de chauffe (dont $83 \mathrm{~m}^{2}$ pour la surchauffe) et une surface de grille de $4 \mathrm{~m}^{2} 600$.

Un câble souterrain relie le tableau de distribution de ce groupe à vapeur avec celui de l'usine hydro-électrique et peut être à volonté connecté avec barres collectrices princi-. pales ou avec les barres auxiliaires. Cette unité de réserve est utilisée aux époques des hautes eaux lorsque la hauteur de chute est réduite, pendant l'hiver quand les glaces encombrent le canal ou enfin aux moments où les charges maxirua des réseaux de force èt de lumièré coinncident.

Réseau de distribution. - De l'usine partent du côté Suisse deux câbles souterrains sè rendant à un poste de distribution, et du côté de la rive droite du Rhin cinq autres câbles se rendànt à un second poste de distribution. De ces postes, le courant est conduit par conducteurs souterrains et par canalisatioṇs aériennes aux différentes sousstations de transformation réparties sur le réseau de distribution. Dans ces sous-stations la tension est abaissée de 6800 volts à 500 et 220 volts qui sont les tensions des réseaux secondaires. 28 sous-stations d'une capacité totale de 3800 KVA transforment le courant à 500 volts, et 23 autres sous-stations d'une capacité totale de $970 \mathrm{KVA}$ le transforment à 220 volts; soit au total 5 I sous-stations d'une capacité de $477^{\circ}$ KVA.

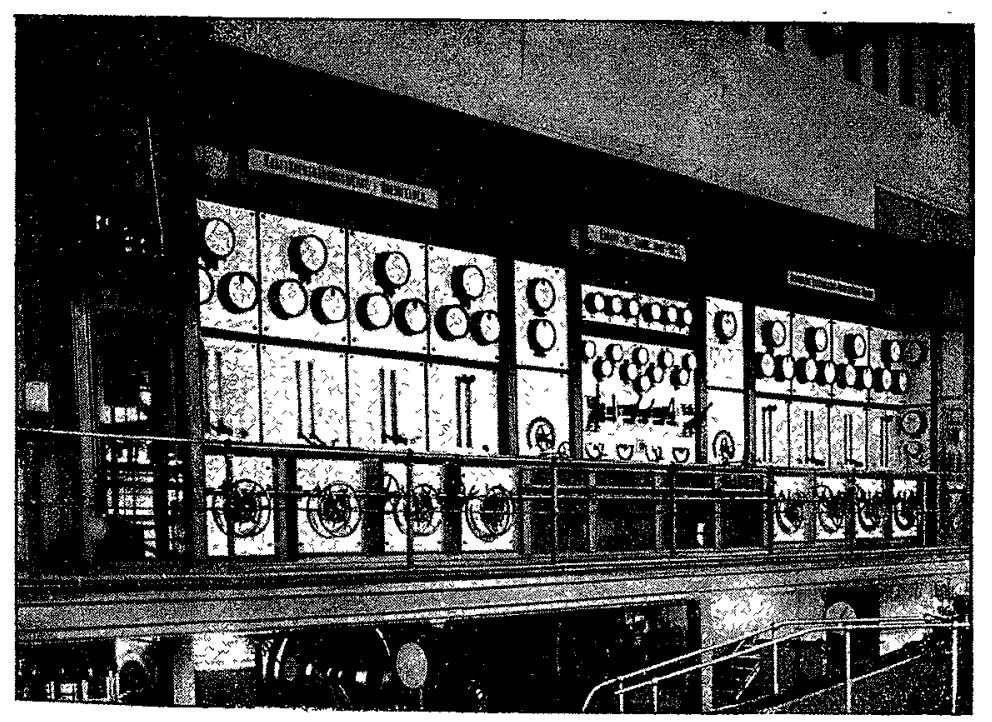

Vue générale du tableau de distribution.

En plus de ces postes de transformateurs, les réseaux possèdent encore les quatre sous-stations suivantes: Rheinfelden, $175 \mathrm{Kw}$; Schopfheim, $86 \mathrm{Kw}$; Wehr, $46 \mathrm{Kw}$ : Zörrach, $200 \mathrm{Kw}$. Dans ces dernières, le courant triphasé est converti en courant continúà l'aide de commutatrices et emmagasiné dans des batteries d'accumulateurs.

Le courant ainsi distribué est pour la plus grande partie employé sous forme de force motrice par des tissages, teintureries, imprimeries, brasseries, ateliers de construction de machines et tourneries sur bois, fabriques de pâtes de bois, etc., ainsi que par la petite industrie virant de l'atelier familial, très répandue dans ce pays. Le courant pour lumière dont la consommation augmente chaque jour est fourni par les quatre dernières sous-stations. Dernièrement, la ville de Bâle est devenue i'un des abonnés de ce réseau pour $2000 \mathrm{HP}$ destinés à l'éclairage, aux tramways et à divers établissements industriels.

Création d'une nouvelle usine. - Une nouvelle et très importante installation, celle de "Elektra SackingenWaldshut ", va être mise en marche l'année prochaine. Sa création est due à une grande association de fabricants de rubans de soie comprenant plus de 30 communautés, qui veut, avec l'aide du Gouvernement, développer l'usage de la commande électrique des métiers de tissage pour conserver dans la région l'industrie du petit atelier familial.

La fourniture d'énergje à cette entreprise, avec celle demandée par la ville de Bâle, sont capables à elles seules d'absorber toute la puissance disponible de l'usine de Rheinfelden. Aussi cette usine, d'accord avec la ville, ontelles décidé l'aménagement d'une nouvelle installation hydraulique à 5 kilomètres en aval de la station actuelle. La demande de concession qui suit son cours doit recevoir son exécution avant un an. Un barrage constitué par des vannes mobiles sera établi sur le Rhin pour alimenter deux usines d'une puissance de i 5 ooo HP, l'une sur la rive Suisse, pour le transport de force de Rheinfelden, et l'autre sur la rive Badoise, pour le transport d'énergie à Bâle. On trouve, pour cette création, des terrains sur les deux rives du fleuve, dans une situation particulièrement avantageuse, tant sous le rapport de l'accès pour les industries qui voudront s'y développer qu'au point de vue des conditions d'achat.

En attendant l'installation de ce nouvel établissement, l'entreprise en question a conclu avec une grande usine (Beznau), située à proximité, un contrat de location de courant. Ce courant doit être fourni à Rheinfelden sous une tension de 25000 volts ; là il sera transformé à la tension de l'usine pour être distribué dans le réseau avec le courant provenant de l'usine elle-même. Il sera possible, dans ces conditions, de répondre aux demandes d'énergie électrique pendant la période même de construction.

Nous reviendrons plus complètement sur ces intéressantes installations lorsqu'elles seront terminées.

\section{Paul Bergeon, Ancien Elève de l'Ecole Supérieure d'Electricité.}

\section{DE L'INTERVENTION DE L'ÉLECTROCHIMIE}

\section{DANS LA MÉTALLURGIE DU PLOMB}

\section{(Ancienne méthode d'exploitation de Pontgibaud) (Fin)}

\section{VII. - RAFFINAGE DE L'ARGENT}

Argent éclair venant de la désargentation par la vapeur d'eau. - A Pontgibaud, on raffinair l'argent dans des creusets en plombagine, préparée par le procédé Morgan, ayant leurs couvercles en même matière; ils étaient placés dans des fours à vent alimentés d'air par une galerie souterraine et desservis par une cheminée commune.

Il y avait deux fourneaux disposés dans un petit atelier représentant une chambre de $3 \mathrm{~m}_{00}$ de large environ et de 4 ou 5 moo de longueur. Les fours étaient adossés au mur 